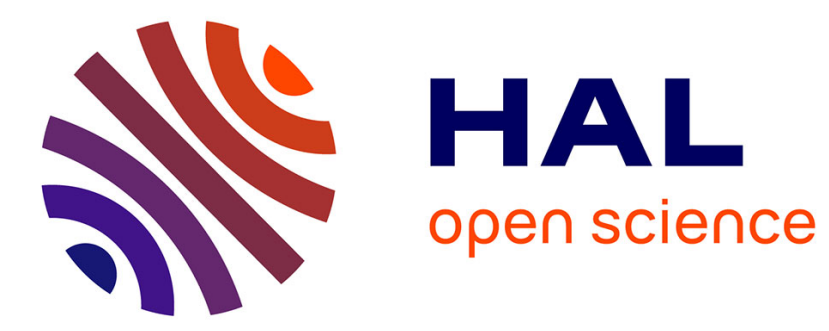

\title{
Etude de la variation de la différence d'énergie entre les deux premières bandes de conduction du GaSb avec la pression hydrostatique
}

G. Bougnot, M. Averous, J. Calas

\section{- To cite this version:}

G. Bougnot, M. Averous, J. Calas. Etude de la variation de la différence d'énergie entre les deux premières bandes de conduction du GaSb avec la pression hydrostatique. Journal de Physique, 1970, 31 (1), pp.73-77. 10.1051/jphys:0197000310107300 . jpa-00206880

\section{HAL Id: jpa-00206880 https://hal.science/jpa-00206880}

Submitted on 1 Jan 1970

HAL is a multi-disciplinary open access archive for the deposit and dissemination of scientific research documents, whether they are published or not. The documents may come from teaching and research institutions in France or abroad, or from public or private research centers.
L'archive ouverte pluridisciplinaire HAL, est destinée au dépôt et à la diffusion de documents scientifiques de niveau recherche, publiés ou non, émanant des établissements d'enseignement et de recherche français ou étrangers, des laboratoires publics ou privés. 


\title{
ÉTUDE DE LA VARIATION DE LA DIFFÉRENGE D'ÉNERGIE ENTRE LES DEUX PREMIËRES BANDES DE GONDUGTION DU GaSb AVEG LA PRESSION HYDROSTATIQUE
}

\author{
Par G. BOUGNOT, M. AVEROUS et J. CALAS, \\ Gentre d'Études d'Électronique des Solides de Montpellier (associé au C.N.R.S.). \\ (Reçu le 10 avril 1969, révisé le 15 juillet 1969.)
}

\begin{abstract}
Résumé. - Nous développons la théorie de la variation de $\Delta E$, différence d'énergie entre les bandes de conduction (000) et (111) d'un semiconducteur comme GaSb, avec la pression.

Pour cela, nous tenons compte des variations de population et des variations de mobilité de chaque bande lorsque le niveau de Fermi et les masses effectives varient.

Nous appliquons les résultats théoriques obtenus au GaSb et nous confirmons que les deux bandes $(000)$ et (111) se rapprochent lorsque la pression augmente [2], [11], [14], [15]. La valeur de $\frac{\mathrm{d} \Delta E}{\mathrm{~d} P}$ trouvée est de $5,5 \times 10^{-6} \mathrm{eV} /$ bar et est voisine de celle trouvée par Kosicki [9], [16] qui donne $5 \times 10^{-6} \mathrm{eV} /$ bar, en négligeant les variations de mobilité.
\end{abstract}

Abstract. - We develop the theory of the variations with hydrostatic pressure of $\Delta E$, the energy difference between the conduction bands (000) and (111) of a semiconductor such as GaSb.

We take into account the variations of population and mobility of each band when the Fermi level and effective masses change.

We apply the theoretical results to GaSb and we show that the two bands $(000)$ and (111) get nearer when pressure increases. We find $\frac{\mathrm{d} \Delta E}{\mathrm{~d} P}$ equal to $5.5 \times 10^{-6} \mathrm{eV} / \mathrm{bar}$; this value is closed to Kosicki's result which is $5 \times 10^{-6} \mathrm{eV} / \mathrm{bar}$, if the variations of mobility are neglected.

I. Introduction. - Le tenseur de piézorésistance a d'où : été défini [1] par la relation :

$$
\frac{\partial \rho_{i j}}{\rho_{i j}}=\sum_{k, l} \pi_{i j k l} K_{k l}
$$

$\partial \rho$ est la variation de la résistivité du matériau par application d'une contrainte; $\pi$ est le tenseur de piézorésistance; $K$ est le tenseur de contrainte.

Comme $\rho$ et $K$ sont des tenseurs symétriques, on peut les considérer comme des vecteurs à six composantes et l'on obtient :

$$
\frac{\partial \rho_{\omega}}{\rho_{\omega}}=\sum_{\lambda} \pi_{\omega \lambda} K_{\lambda} \quad(\omega \text { et } \lambda \text { variant de } 1 \text { à } 6) \text {. }
$$

Pour un matériau de symétrie cubique $\rho_{1}=\rho_{2}=\rho_{3}$, $\rho_{4}=\rho_{5}=\rho_{6}=0$ et le tenseur de piézorésistance se réduit à trois coefficients indépendants $\pi_{11}, \pi_{12}, \pi_{44}$.

Dans le cas d'une pression hydrostatique :

$$
K=-1 . P \quad(1 \text { est le tenseur unité). }
$$

Nous appliquons des pressions suffisamment faibles pour que $\rho$ varie linéairement avec $P$, de telle sorte que le coefficient de piézorésistance obtenu sous pression hydrostatique est :

$$
\pi_{\mathrm{P}}=-\frac{1}{\rho} \frac{\partial \rho}{\partial P}
$$

$\pi_{\mathrm{P}}$ est relié aux coefficients indépendants du tenseur de piézorésistance par la relation :

$$
\pi_{\mathrm{P}}=\pi_{11}+2 \pi_{12}
$$

$$
\pi_{11}+2 \pi_{12}=\frac{1}{\sigma} \frac{\partial \sigma}{\partial P} .
$$

Dans le cas d'une structure à deux bandes de conduction, telle qu'elle se présente dans le GaSb :

$$
\sigma=n_{1} e \mu_{1}+n_{2} e \mu_{2}
$$

$\mu_{1}$ et $\mu_{2}$ : mobilités des bandes 1 et 2 ,

$n_{1}$ et $n_{2}$ : populations des bandes 1 et 2 ,

donc :

$$
\mathrm{d} \sigma=e\left(\mu_{1} \mathrm{~d} n_{1}+\mu_{2} \mathrm{~d} n_{2}\right)+e\left(n_{1} \mathrm{~d} \mu_{1}+n_{2} \mathrm{~d} \mu_{2}\right) .
$$

Nous allons traiter séparément ces deux termes.

II. Piézorésistance due à la variation des populations. - Nous employons la statistique de Fermi-Dirac, posons :

$$
\begin{aligned}
& n_{1}=4 \pi\left[\frac{2 k T m_{1}^{*}}{h^{2}}\right]^{3 / 2} F_{1 / 2}\left(\eta_{1}\right) \\
& n_{2}=4 \pi\left[\frac{2 k T m_{2}^{*}}{h^{2}}\right]^{3 / 2} F_{1 / 2}\left(\eta_{2}\right)
\end{aligned}
$$

$m_{1}^{*}$ et $m_{2}^{*}$ sont les masses effectives des bandes 1 et 2 . Comme la bande 2 située dans la direction (111) est une bande multivallée avec des surfaces d'énergie ellipsoïdales :

$$
m_{2}^{* 3 / 2}=N_{\nu} m_{\mathrm{L}}^{* 1 / 2} m_{\mathrm{T}}^{*}
$$

$N_{\nu}$ est le nombre de vallées; $m_{\mathrm{T}}$, masse effective transversale; $m_{\mathrm{L}}$, masse effective longitudinale. 


$$
\begin{gathered}
\eta_{1}=\frac{E_{\mathrm{F}}-E_{\mathrm{c}_{1}}}{k T} ; \quad \eta_{2}=\frac{E_{\mathrm{F}}-E_{\mathrm{c}_{2}}}{k T} \\
F_{1 / 2}(\eta)=\int_{0}^{\infty} \frac{\varepsilon^{1 / 2} \mathrm{~d} \varepsilon}{1+\mathrm{e}^{\varepsilon-\eta}}
\end{gathered}
$$

$E_{\mathrm{F}}$ est l'énergie du niveau de Fermi; $E_{\mathrm{c}_{1}}$, l'énergie $\mathrm{du}$ bas de la première bande de conduction; $E_{\mathrm{c}_{2}}$, l'énergie du bas de la deuxième bande de conduction.

II.1. Effet dû A LA VARIATion de $\eta_{1}$ ET DE $\eta_{2}$. Nous supposons que le nombre total de porteurs dans la bande de conduction n'est pas modifié par la pression :

$$
-\frac{\mathrm{d} n_{1}}{\mathrm{~d} P}=\frac{\mathrm{d} n_{2}}{\mathrm{~d} P} \text {. }
$$

Si maintenant on tient compte uniquement de la variation de la différence d'énergie $\Delta E$ entre les bandes $\left(\Delta E=E_{\mathrm{c}_{2}}-E_{\mathrm{c}_{1}}\right)$, on a :

$$
\begin{gathered}
\left.\left.\left.\frac{\partial \sigma}{\partial P}\right)_{m^{*}, \mu}=e \mu_{1} \frac{\partial n_{1}}{\partial P}\right)_{m_{1}^{*}}+e \mu_{2} \frac{\partial n_{2}}{\partial P}\right)_{m_{2}^{*}} \\
\left.\frac{1}{\sigma} \frac{\partial \sigma}{\partial P}\right)_{m^{*}, \mu}=\frac{\left.\left.\frac{\partial n_{1}}{\partial P}\right)_{m_{1}^{*}}+b \frac{\partial n_{2}}{\partial P}\right)_{m_{2}^{*}}}{n_{1}(1+\alpha b)}
\end{gathered}
$$$$
\text { avec : } \quad \alpha=\frac{n_{2}}{n_{1}} ; \quad b=\frac{\mu_{2}}{\mu_{1}} \text {. }
$$

Explicitons $\left.\frac{\partial n_{1}}{\partial P}\right)_{m_{1}^{*}}$ à partir des équations (1). Posons :

$$
\begin{gathered}
C_{1}=4 \pi\left[\frac{2 k T m_{1}^{*}}{h^{2}}\right]^{3 / 2} ; \quad C_{2}=4 \pi\left[\frac{2 k T m_{2}^{*}}{h^{2}}\right]^{3 / 2} \\
\left.\frac{\partial n_{1}}{\partial P}\right)_{m_{1}^{*}}=C_{1} \frac{\mathrm{d} F_{1 / 2}\left(\eta_{1}\right)}{\mathrm{d} \eta_{1}} \frac{\mathrm{d} \eta_{1}}{\mathrm{~d} P} \\
F_{1 / 2}(\eta)=\Gamma_{3 / 2} \mathscr{F}_{1 / 2}(\eta)
\end{gathered}
$$

McDougall et Stoner [17] ont montré que :

$$
\begin{gathered}
\frac{\mathrm{d} F_{1 / 2}(\eta)}{\mathrm{d} \eta}=\Gamma_{3 / 2} \mathscr{F}_{-1 / 2}(\eta) ; \quad \mathscr{F}_{-1 / 2}(\eta)=\frac{1}{\Gamma_{1 / 2}} F_{-1 / 2}(\eta) \\
\frac{\mathrm{d} F_{1 / 2}(\eta)}{\mathrm{d} \eta}=\frac{\Gamma_{3 / 2}}{\Gamma_{1 / 2}} F_{-1 / 2}(\eta) ; \quad \Gamma_{1 / 2}=\sqrt{\pi}, \Gamma_{3 / 2}=\frac{\sqrt{\pi}}{2} \\
\frac{\mathrm{d} F_{1 / 2}(\eta)}{\mathrm{d} \eta}=\frac{F_{-1 / 2}(\eta)}{2} .
\end{gathered}
$$

Portons cette expression dans l'équation (3) :

$$
\left.\frac{\partial n_{1}}{\partial P}\right)_{m_{1}^{*}}=C_{1} \frac{F_{-1 / 2}\left(\eta_{1}\right)}{2} \frac{\mathrm{d} \eta_{1}}{\mathrm{~d} P}
$$

d'où : $\left.\quad \frac{1}{\sigma} \frac{\mathrm{d} \sigma}{\mathrm{d} P}\right)_{m^{*}, \mu}=\frac{1}{2} \frac{1-b}{1+\alpha b} \frac{F_{-1 / 2}\left(\eta_{1}\right)}{F_{1 / 2}\left(\eta_{1}\right)} \frac{\mathrm{d} \eta_{1}}{\mathrm{~d} P}$.

Explicitons $\frac{\partial n_{1}}{\partial P}$ et posons :

$$
\Delta=\frac{\Delta E}{k T}
$$

donc $: \eta_{1}-\eta_{2}=\Delta$.
Avec :

$$
\left.\left.\frac{\partial n_{1}}{\partial P}\right)_{m_{1}^{*}}=-\frac{\partial n_{2}}{\partial P}\right)_{m_{2}^{*}} \text { et } \quad \frac{\mathrm{d} \eta_{1}}{\mathrm{~d} P}-\frac{\mathrm{d} \eta_{2}}{\mathrm{~d} P}=\frac{\mathrm{d} \Delta}{\mathrm{d} P}
$$

on obtient :

$$
\frac{\partial \eta_{1}}{\mathrm{~d} P}=\frac{\frac{\mathrm{d} \Delta}{\mathrm{d} P}}{1+\frac{1}{\alpha} \frac{F_{1 / 2}\left(\eta_{2}\right)}{F_{-1 / 2}\left(\eta_{2}\right)} \frac{F_{-1 / 2}\left(\eta_{1}\right)}{F_{1 / 2}\left(\eta_{1}\right)}}
$$

Portons (5) dans (4), on obtient :

$$
\begin{aligned}
\left.\frac{1}{\sigma} \frac{\mathrm{d} \sigma}{\mathrm{d} P}\right)_{m^{*}, \mu}= & \frac{1}{2} \frac{1-b}{1+\alpha b} \\
& \frac{\alpha \frac{F_{-1 / 2}\left(\eta_{2}\right)}{F_{+1 / 2}\left(\eta_{2}\right)}}{1+\alpha \frac{F_{1 / 2}\left(\eta_{1}\right)}{F_{-1 / 2}\left(\eta_{1}\right)} \frac{F_{-1 / 2}\left(\eta_{2}\right)}{F_{1 / 2}\left(\eta_{2}\right)}} \frac{1}{k T} \frac{\mathrm{d} \Delta E}{\mathrm{~d} P} .
\end{aligned}
$$

II.2. EFFET DÛ A LA VARIATION DE LA MASSE EFFEGTIVE AVEG LA PRESSION. - Dans le cas de la bande 1 qui est une bande isotrope, située au centre de la première zone de Brillouin, on admet que [2] :

$$
m_{1}^{*} \propto E_{\mathrm{g}_{1}}
$$

$E_{\mathrm{g}_{1}}=$ largeur de la bande interdite au centre de la première zone de Brillouin.

$$
\begin{gathered}
\left.\frac{\partial n_{1}}{\partial P}\right)_{n_{1}}=4 \pi\left[\frac{2 k T}{h^{2}}\right]^{3 / 2} F_{1 / 2}\left(\eta_{1}\right) \frac{3}{2} m_{1}^{* 1 / 2} \frac{\mathrm{d} m_{1}^{*}}{\mathrm{~d} P} \\
\left.\frac{1}{n_{1}} \frac{\partial n_{1}}{\partial P}\right)_{n_{1}}=\frac{3}{2} \frac{1}{m_{1}^{*}} \frac{\mathrm{d} m_{1}^{*}}{\mathrm{~d} P}=\frac{3}{2} \frac{1}{E_{\mathrm{g}_{1}}} \frac{\mathrm{d} E_{\mathrm{g}_{1}}}{\mathrm{~d} P} .
\end{gathered}
$$

Dans le cas de la bande 2, qui est une bande multivallée, les calculs de structure de bande de Bordure [3] montrent que seule la masse transversale $m_{\mathrm{T}}$ est sensible à $E_{\mathrm{g}_{2}}$ et que $m_{\mathrm{T}} \propto E_{\mathrm{g}_{2}}$ :

$$
\begin{gathered}
\left.\frac{\partial n_{2}}{\partial P}\right)_{n_{2}}=4 \pi\left[\frac{2 k T}{h^{2}}\right]^{3 / 2} F_{1 / 2}\left(\eta_{2}\right) N_{\mathrm{v}} m_{\mathrm{L}}^{*} 1 / 2 \frac{\mathrm{d} m_{\mathrm{T}}}{\mathrm{d} P} \\
\left.\frac{1}{n_{2}} \frac{\partial n_{2}}{\partial P}\right)_{n_{2}}=\frac{1}{m_{\mathrm{T}}^{*}} \frac{\mathrm{d} m_{\mathrm{T}}^{*}}{\mathrm{~d} P}=\frac{1}{E_{\mathrm{g}_{2}}} \frac{\mathrm{d} E_{\mathrm{g}_{2}}}{\mathrm{~d} P} .
\end{gathered}
$$

Des expressions (7) et (8) nous déduisons la contribution à la piézorésistance, de la variation des populations due à la variation de la masse effective avec la pression :

$$
\begin{gathered}
\left.\frac{1}{\sigma} \frac{\partial \sigma}{\partial P}\right)_{n}=\frac{\mu_{1} n_{1} \frac{3}{2} \frac{1}{E_{\mathrm{g}_{1}}} \frac{\mathrm{d} E_{\mathrm{g}_{1}}}{\mathrm{~d} P}+\mu_{2} n_{2} \frac{1}{E_{\mathrm{g}_{2}}} \frac{\mathrm{d} E_{\mathrm{g}_{2}}}{\mathrm{~d} P}}{\mu_{1} n_{1}+\mu_{2} n_{2}} \\
\left.\frac{1}{\sigma} \frac{\partial \sigma}{\partial P}\right)_{\eta}=\frac{\overline{2} \frac{1}{E_{\mathrm{g}_{1}}} \frac{\mathrm{d} E_{\mathrm{g}_{1}}}{\mathrm{~d} P}+\alpha b \frac{1}{E_{\mathrm{g}_{2}}} \frac{\mathrm{d} E_{\mathrm{g}_{2}}}{\mathrm{~d} P}}{1+\alpha b} .
\end{gathered}
$$

La contribution totale à la piézorésistance due à la variation des populations est obtenue à partir des équations (8) et (9) :

$$
\left.\pi_{11}+2 \pi_{12}\right)_{\mu=\mathrm{Cte}}=\frac{1}{2 k T} \frac{1-b}{1+\alpha b} \frac{\alpha \frac{F_{-1 / 2}\left(\eta_{2}\right)}{F_{1 / 2}\left(\eta_{2}\right)} \frac{\mathrm{d} \Delta E}{\mathrm{~d} P}}{1+\alpha \frac{F_{1 / 2}\left(\eta_{1}\right)}{F_{-1 / 2}\left(\eta_{1}\right)} \frac{F_{-1 / 2}\left(\eta_{2}\right)}{F_{1 / 2}\left(\eta_{2}\right)}}+\frac{\frac{3}{2} \frac{1}{E_{\mathrm{g}_{1}}} \frac{\mathrm{d} E_{\mathrm{g}_{1}}}{\mathrm{~d} P}+\alpha b \frac{1}{E_{\mathrm{g}_{2}}} \frac{\mathrm{d} E_{\mathrm{g}_{2}}}{\mathrm{~d} P}}{1+\alpha b} .
$$


III. Piézorésistance due à la variation des mobilités. - L'étude des mobilités en fonction de la température effectuée par J. L. Robert [4] montre qu'à 300 KK la température est suffisamment élevée pour que toutes les impuretés soient ionisées, et les seuls modes de diffusion à considérer sont les modes acoustiques, d'où une variation de $\mu$ en $T^{-3 / 2}$.

Le temps de relaxation de ce processus a été calculé à partir de la théorie du potentiel de déformation [5], [6], [7] :

$$
\tau=\frac{\pi \hbar^{4}}{\sqrt{2} E_{1}^{2}} \frac{\mathrm{d} u_{1}^{2}}{m^{* 3 / 2}(k T)^{3 / 2}}
$$

$d=$ densité du solide,

$u_{1}=$ vitesse de l'onde acoustique longitudinale,

$E_{1}=$ variation de l'énergie du bord de la bande pour une dilatation unité.

La mobilité de conductivité est donnée par [8] :

$\mu(\eta)=-\frac{2}{3} \frac{e \tau}{m^{*}} \frac{F_{0}(\eta)}{F_{1 / 2}(\eta)}=-\frac{\sqrt{2}}{3 E_{1}^{2}} \frac{\pi e \hbar^{4} \mathrm{~d} u_{1}^{2}}{m^{*} 5 / 2(k T)^{3 / 2}} \frac{F_{0}(\eta)}{F_{1 / 2}(\eta)}$.

III.1. EFFet dÛ A LA VARIATION DE LA Mobilité $\mu_{1}$ DANS LA PREMIÈRE BANDE. — Dans le cas de la première bande, la masse effective est isotrope :

$\mu_{1}=C \frac{F_{0}\left(\eta_{1}\right)}{F_{1 / 2}\left(\eta_{1}\right)} \frac{1}{m_{1}^{* 5 / 2}} \quad$ avec $\quad C=-\frac{\sqrt{2}}{3 E_{1}^{2}} \frac{\pi e \hbar^{4} \mathrm{~d} u_{1}^{2}}{(k T)^{3 / 2}}$.
III.1.1. Variation de la mobilité avec la masse effective. - En rappelant que l'on admet que $m_{1} \propto E_{\mathrm{g}_{1}}$, nous avons donc :

$$
\left.\frac{1}{\mu_{1}} \frac{\partial \mu_{1}}{\partial P}\right)_{r_{1}}=-\frac{5}{2 m_{1}^{*}} \frac{\mathrm{d} m_{1}^{*}}{\mathrm{~d} P}=-\frac{5}{2} \frac{\mathrm{d} E_{\mathrm{g}_{1}}}{\mathrm{~d} P} \frac{1}{E_{\mathrm{g}_{1}}} .
$$

III .1.2. Variation de la mobilité avec $\eta_{1}$. — Nous avons vu que :

$$
\frac{\mathrm{d} F_{1 / 2}(\eta)}{\mathrm{d} \eta}=\frac{1}{2} F_{-1 / 2}(\eta)
$$

de même :

$$
\begin{gathered}
\frac{\mathrm{d} F_{0}(\eta)}{\mathrm{d} \eta}=F_{-1}(\eta) \quad \text { car } \quad \Gamma_{1}=\Gamma_{0}=1 \\
\left.\frac{1}{\mu_{1}} \frac{\partial \mu_{1}}{\partial P}\right)_{m_{1}^{*}}=\left[\frac{F_{-1 / 2}\left(\eta_{1}\right)}{F_{0}\left(\eta_{1}\right)}-\frac{F_{-1 / 2}\left(\eta_{1}\right)}{2 F_{1 / 2}\left(\eta_{1}\right)}\right] \frac{\mathrm{d} \eta_{1}}{\mathrm{~d} P} .
\end{gathered}
$$

Remplaçons $\frac{\mathrm{d} \eta_{1}}{\mathrm{~d} P}$ par l'expression trouvée [5] :

$$
\begin{aligned}
& \left.\frac{1}{\mu_{1}} \frac{\partial \mu_{1}}{\partial P}\right)_{m_{1}^{*}} \\
& =\left[\frac{F_{-1}\left(\eta_{1}\right)}{F_{0}\left(\eta_{1}\right)}-\frac{F_{-1 / 2}\left(\eta_{1}\right)}{2 F_{1 / 2}\left(\eta_{1}\right)}\right] \frac{\frac{\mathrm{d} \Delta}{\mathrm{d} P}}{1+\alpha^{-1} \frac{F_{1 / 2}\left(\eta_{2}\right)}{F_{-1 / 2}\left(\eta_{2}\right)} \frac{F_{-1 / 2}\left(\eta_{1}\right)}{F_{1 / 2}\left(\eta_{1}\right)}} .
\end{aligned}
$$

La variation totale de la mobilité $\mu_{1}$ est donnée par les équations (12) et (13) :

$$
\frac{\mathrm{d} \mu_{1}}{\mathrm{~d} P}=\left\{-\frac{5}{2} \frac{\mathrm{d} E_{\mathrm{g}_{1}}}{\mathrm{~d} P} \frac{1}{E_{\mathrm{g}_{1}}}+\left[\frac{F_{-1}\left(\eta_{1}\right)}{F_{0}\left(\eta_{1}\right)}-\frac{F_{-1 / 2}\left(\eta_{1}\right)}{2 F_{1 / 2}\left(\eta_{1}\right)}\right] \frac{\mathrm{d} \Delta E}{\mathrm{~d} P} \frac{1}{1+\alpha \frac{F_{1 / 2}\left(\eta_{2}\right)}{F_{-1 / 2}\left(\eta_{2}\right)} \frac{F_{-1 / 2}\left(\eta_{1}\right)}{F_{1 / 2}\left(\eta_{1}\right)}} \frac{1}{k T}\right\} \mu_{1} .
$$

III.2. EfFet dÛ A LA VARIATION DE LA Mobilité $\mu_{2}$ DE LA DEUXIÈmE BANDE. - La deuxième bande est multivallée, on va donc remplacer $m_{2}^{*-5 / 2}$ par $\left(m_{\mathrm{L}}^{*} m_{\mathrm{T}}^{* 2}\right)^{-1 / 2} \frac{1}{3}\left(m_{\mathrm{L}}^{*-1}+2 m_{\mathrm{T}}^{*-1}\right)$.

III.2.1. Variation de $\mu_{2}$ avec la masse effective :

$$
\begin{gathered}
\mu_{2}=\frac{C}{\left(m_{\mathrm{T}}^{*} m_{\mathrm{T}}^{* 2}\right)^{1 / 2}} \frac{1}{3}\left[\frac{1}{m_{\mathrm{L}}^{*}}+\frac{2}{m_{\mathrm{T}}^{*}}\right] \frac{F_{0}\left(\eta_{2}\right)}{F_{1 / 2}\left(\eta_{2}\right)} \\
\left.\frac{1}{\mu_{2}} \frac{\partial \mu_{2}}{\partial P}\right)_{n_{2}}=-\frac{\frac{4}{m_{\mathrm{T}}^{*}}+\frac{1}{m_{\mathrm{L}}^{*}}}{\frac{2}{m_{\mathrm{T}}^{*}}+\frac{1}{m_{\mathrm{T}}^{*}}} \frac{m_{\mathrm{T}}^{*}}{}
\end{gathered}
$$

puisque seule $m_{\mathrm{T}}^{*}$ varie avec la largeur de la bande interdite.

$$
\left.\frac{1}{\mu_{2}} \frac{\partial \mu_{2}}{\partial P}\right)_{\eta_{2}}=-\frac{1}{E_{\mathrm{g}_{2}}} \frac{\mathrm{d} E_{\mathrm{g}_{2}}}{\mathrm{~d} P} \frac{1+4 K}{1+2 K}
$$

où $K$ représente l'anisotropie des ellipsoïdes d'énergie.

III.2.2. Variation de $\mu_{2}$ avec $\eta_{2}$. - Un traitement analogue au paragraphe III.1.1 nous donne :

$$
\begin{gathered}
\left.\frac{1}{\mu_{2}} \frac{\partial \mu_{2}}{\partial P}\right)_{m_{2}^{*}}=\left[\frac{F_{-1}\left(\eta_{2}\right)}{F_{0}\left(\eta_{2}\right)}-\frac{F_{-1 / 2}\left(\eta_{2}\right)}{2 F_{1 / 2}\left(\eta_{2}\right)}\right] \frac{\mathrm{d} \eta_{2}}{\mathrm{~d} P} \\
\frac{\mathrm{d} \eta_{2}}{\mathrm{~d} P}=\frac{\mathrm{d} \eta_{1}}{\mathrm{~d} P}-\frac{\mathrm{d} \Delta}{\mathrm{d} P} \\
\frac{\mathrm{d} \eta_{2}}{\partial P}=-\frac{\mathrm{d} \Delta}{\mathrm{d} P} \frac{1}{1+\alpha \frac{F_{1 / 2}\left(\eta_{1}\right)}{F_{-1 / 2}\left(\eta_{1}\right)} \frac{F_{-1 / 2}\left(\eta_{2}\right)}{F_{1 / 2}\left(\eta_{2}\right)}}
\end{gathered}
$$

donc :

$$
\left.\frac{1}{\mu_{2}} \frac{\partial \mu_{2}}{\partial P}\right)_{m_{2}^{*}}=-\left[\frac{F_{-1}\left(\eta_{2}\right)}{F_{0}\left(\eta_{2}\right)}-\frac{F_{-1 / 2}\left(\eta_{2}\right)}{2 F_{1 / 2}\left(\eta_{2}\right)}\right] \frac{\mathrm{d} \Delta E}{\mathrm{~d} P} \frac{1}{k T} \frac{1}{1+\alpha \frac{F_{1 / 2}\left(\eta_{1}\right)}{F_{-1 / 2}\left(\eta_{1}\right)} \frac{F_{-1 / 2}\left(\eta_{2}\right)}{F_{1 / 2}\left(\eta_{2}\right)}}
$$

Des équations (15) et (16) on obtient la variation totale de la mobilité :

$$
\frac{\mathrm{d} \mu_{2}}{\partial P}=\left\{-\frac{1}{E_{\mathrm{g}_{2}}} \frac{\mathrm{d} E_{\mathrm{g}_{2}}}{\mathrm{~d} P} \frac{1+4 K}{1+2 K}-\left[\frac{F_{-1}\left(\eta_{2}\right)}{F_{0}\left(\eta_{2}\right)}-\frac{F_{-1 / 2}\left(\eta_{2}\right)}{2 F_{1 / 2}\left(\eta_{2}\right)}\right] \frac{1}{1+\alpha \frac{F_{1 / 2}\left(\eta_{11}\right)}{F_{1 / 2}\left(\eta_{2}\right)} \frac{F_{-1 / 2}\left(\eta_{2}\right)}{F_{-1 / 2}\left(\eta_{1}\right)}} \frac{1}{k T} \frac{\mathrm{d} \Delta E}{\mathrm{~d} P}\right\} \mu_{2} .
$$


Des équations (14) et (17) nous pouvons calculer la contribution à la piézorésistance provenant de la variation des mobilités avec la pression :

$$
\begin{aligned}
\frac{1}{\sigma} \frac{\mathrm{d} \sigma}{\mathrm{d} P}=(1 & +\alpha b)^{-1}\left\{-\frac{5}{2} \frac{\mathrm{d} E_{\mathrm{g}_{1}}}{\mathrm{~d} P} \frac{1}{E_{\mathrm{g}_{1}}}+\frac{1}{k T} \frac{\mathrm{d} \Delta E}{\mathrm{~d} P} \frac{\alpha \frac{F_{1 / 2}\left(\eta_{1}\right)}{F_{1 / 2}\left(\eta_{2}\right)} \frac{F_{-1 / 2}\left(\eta_{2}\right)}{F_{-1 / 2}\left(\eta_{1}\right)}}{1+\alpha \frac{F_{1 / 2}\left(\eta_{1}\right)}{F_{-1 / 2}\left(\eta_{1}\right)} \frac{F_{-1 / 2}\left(\eta_{2}\right)}{F_{1 / 2}\left(\eta_{2}\right)}}\left[\frac{F_{-1}\left(\eta_{1}\right)}{F_{0}\left(\eta_{1}\right)}-\frac{F_{-1 / 2}\left(\eta_{1}\right)}{2 F_{1 / 2}\left(\eta_{1}\right)}\right]\right. \\
& \left.+\left(-\frac{1}{E_{\mathrm{g}_{2}}} \frac{\mathrm{d} E_{\mathrm{g}_{2}}}{\mathrm{~d} P} \frac{1}{1+2 K}-\left[\frac{F_{-1}\left(\eta_{2}\right)}{F_{0}\left(\eta_{2}\right)} \frac{F_{-1 / 2}\left(\eta_{2}\right)}{2 F_{1 / 2}\left(\eta_{2}\right)}\right] \frac{1}{1+\alpha \frac{F_{1 / 2}\left(\eta_{1}\right)}{F_{-1 / 2}\left(\eta_{1}\right)} \frac{F_{-1 / 2}\left(\eta_{2}\right)}{F_{1 / 2}\left(\eta_{2}\right)}} \frac{1}{k T} \frac{\mathrm{d} \Delta E}{\mathrm{~d} P}\right) \alpha b\right\}
\end{aligned}
$$

Les équations (10) et (18) vont nous donner la piézorésistance totale. En posant que $E_{\mathrm{g}_{2}}-E_{\mathrm{g}_{1}}=\Delta E$, on obtient :

$$
\frac{\mathrm{d} E_{\mathrm{g}_{2}}}{\mathrm{~d} P}=\frac{\mathrm{d} \Delta E}{\mathrm{~d} P}+\frac{\mathrm{d} E_{\mathrm{g}_{1}}}{\mathrm{~d} P}
$$

On obtient pour la piézorésistance totale :

$$
\begin{aligned}
\pi_{11}+2 \pi_{12}=\frac{\mathrm{d} \Delta E}{\mathrm{~d} P}(1+\alpha b)^{-1}\left\{\frac{\alpha b}{E_{\mathrm{g}_{2}}}\left(1-\frac{1+4 K}{1+2 K}\right)+\frac{\alpha}{k T} \frac{1}{1+\alpha \frac{F_{1 / 2}\left(\eta_{1}\right)}{F_{-1 / 2}\left(\eta_{1}\right)} \frac{F_{-1 / 2}\left(\eta_{2}\right)}{F_{1 / 2}\left(\eta_{2}\right)}}\right. \\
\left.\left(\frac{1-b}{2} \frac{F_{-1 / 2}\left(\eta_{2}\right)}{F_{1 / 2}\left(\eta_{2}\right)}+\frac{F_{1 / 2}\left(\eta_{1}\right)}{F_{-1 / 2}\left(\eta_{1}\right)} \frac{F_{-1 / 2}\left(\eta_{2}\right)}{F_{1 / 2}\left(\eta_{2}\right)}\left[\frac{F_{-1}\left(\eta_{1}\right)}{F_{0}\left(\eta_{1}\right)}-\frac{F_{-1 / 2}\left(\eta_{1}\right)}{2 F_{1 / 2}\left(\eta_{1}\right)}\right]-b\left[\frac{F_{-1}\left(\eta_{2}\right)}{F_{0}\left(\eta_{2}\right)}-\frac{F_{-1 / 2}\left(\eta_{2}\right)}{2 F_{1 / 2}\left(\eta_{2}\right)}\right]\right)\right\} \\
+\frac{\mathrm{d} E_{\mathrm{g}_{1}}}{\mathrm{~d} P}(1+\alpha b)^{-1}\left\{-\frac{1}{E_{\mathrm{g}_{1}}}+\frac{\alpha b}{E_{\mathrm{g}_{2}}}\left(1-\frac{1+4 K}{1+2 K}\right)\right\} .
\end{aligned}
$$

IV. Résultats. - Nous avons travaillé sur quatre échantillons de type $n$. Ces échantillons ont été découpés par une usineuse à ultrasons, dans les lingots de GaSb obtenus par tirage (méthode de Czochralski); le GaSb étant naturellement de type $p$, nous avons introduit dans le bain, avant le tirage, du tellure comme impureté donatrice. L'étude de la constante de Hall en fonction de la température montre que nous pouvons considérer tous les donneurs comme ionisés [9] (fig. 1).

La légère décroissance du coefficient de Hall observée pour l'échantillon ayant la constante de Hall la

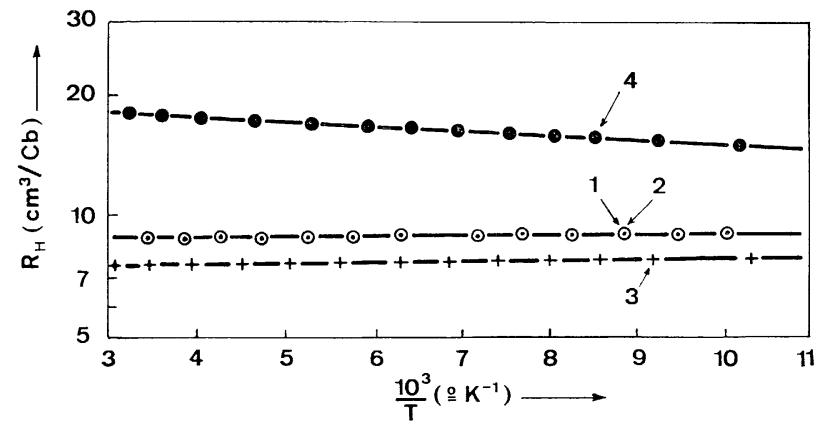

Fig. 1. - Variation de la constante de Hall avec la température.

plus élevée est due au transfert des électrons de la bande (111) à la bande (000) quand la température décroît [9].

Les mesures de piézorésistance, sous pression hydrostatique, ont été effectuées pour des pressions allant de 0 à $4000 \mathrm{~kg} / \mathrm{cm}^{2}$. La chambre d'expérience étant constituée par une enceinte en bronze-béryllium, la pression étant obtenue par l'intermédiaire d'un com- presseur thermique, l'agent transmetteur de pression est l'azote.

Ce montage de mesure de pression hydrostatique nous permet de faire des mesures entre $77 \mathrm{oK}$ et $300 \mathrm{oK}$. Nous l'utilisons dans le présent travail uniquement à $300 \mathrm{oK}$.

Nous travaillons avec un champ de l'ordre de 0,5 tesla, c'est-à-dire dans un domaine où $R_{\mathrm{H}}$ est indépendant du champ.

Dans un modèle à deux bandes de conduction [11], [12], du GaSb, nous pouvons écrire à $300 \mathrm{oK}$ :

$$
R_{\mathrm{H}}=\frac{n_{1} \mu_{1}^{2}+n_{2} \mu_{2}^{2}}{e\left(n_{1} \mu_{1}+n_{2} \mu_{2}\right)^{2}}
$$

car nous prenons $\mu_{\mathrm{H}}=\mu$, puisque $k T$ est de l'ordre de $2,59 \times 10^{-2} \mathrm{eV}$, c'est-à-dire grand devant l'énergie des niveaux de Landau [10].

En prenant à nouveau $\alpha=\frac{n_{2}}{n_{1}}$ et $b=\frac{\mu_{2}}{\mu_{1}}$

$$
R_{\mathrm{H}}=\frac{1}{e} \frac{1}{n_{1}} \frac{1+\alpha b^{2}}{(1+\alpha b)^{2}}
$$

d'après les expressions (1) du paragraphe II, pour un $b$ donné, $R_{\mathrm{H}}$ est une fonction de $\eta_{1}$ seulement, puisque $\eta_{1}$ est relié à $\eta_{2}$ par :

$$
\eta_{1}-\eta_{2}=\frac{\Delta E}{k T}
$$

Si nous nous fixons [13], [14] :

$$
\begin{aligned}
\Delta E & =0,08 \mathrm{eV} \\
m_{1}^{*} & =0,047 m_{\mathrm{e}}
\end{aligned}
$$

$m_{2}^{*}=0,50 m_{\mathrm{e}}=$ masse effective

de densité d'état du deuxième minimum nous pouvons, pour chaque $R_{\mathrm{H}}$ expérimental, calculer $\eta_{1}, \eta_{2}, n_{1}$ et $n_{2}$. 
TABLEAU I

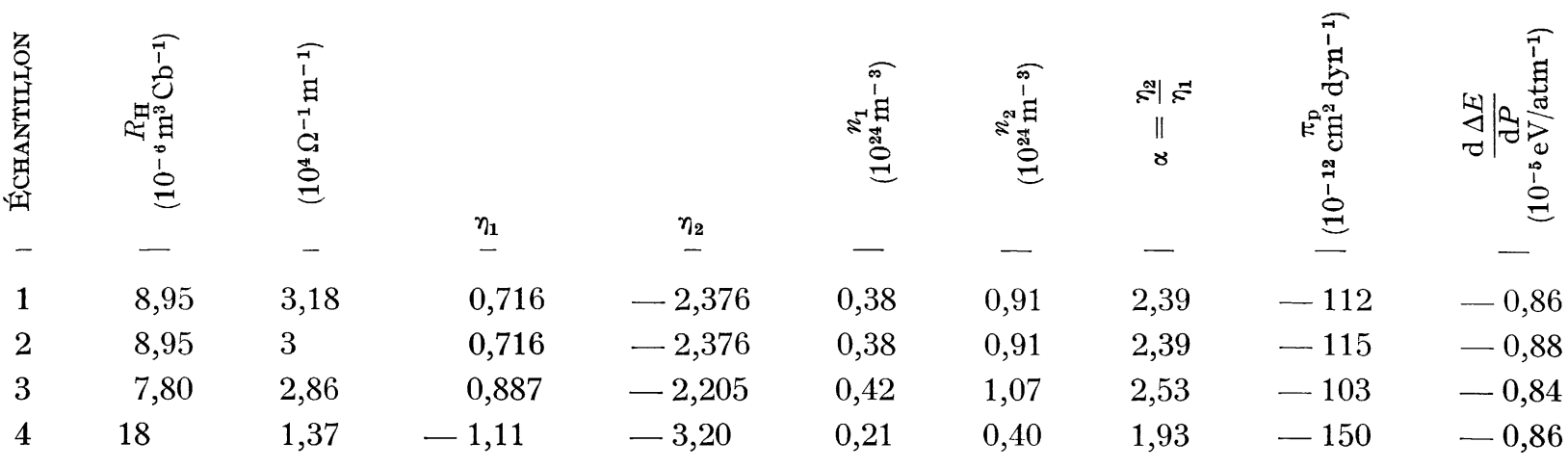

La valeur de $b$ choisie est 0,16 . Cette valeur a été déterminée par un ensemble de mesures en fonction de la température comprenant des mesures de conductivité $\sigma$, de constante de Hall $R_{\mathrm{H}}$, de variation de résistivité sous pression hydrostatique, de variation de résistivité sous pression uniaxiale [15]. Un modèle de calcul autocompatible nous a permis de retenir la valeur de $b=0,16$; cette valeur est en très bon accord avec celle trouvée par d'autres auteurs [4], [18], [14].

Nous avons pris :

$$
E_{\mathrm{g}_{1}} \simeq 0,8 \mathrm{eV}
$$

Nous avons pris comme valeur de $\frac{\mathrm{d} E_{\mathrm{g}_{1}}}{\mathrm{~d} P}$ la valeur de $14,5 \times 10^{-6} \mathrm{eV} /$ bar [9], [16].

Nous avons enfin posé que $\frac{1+4 K}{1+2 K} \simeq 2$ puisque, suivant les auteurs [3], [2], [1] :

$$
10 \leqslant K \leqslant 14 \text {. }
$$

Nous pouvons par conséquent calculer $\frac{\mathrm{d} \Delta E}{\mathrm{~d} P}$.

Les résultats obtenus sont tabulés dans le tableau I. $\frac{\mathrm{d} \Delta E}{\mathrm{~d} P}$ pour tous les échantillons est voisin de $-9 \times 10^{-6} \mathrm{eV} / \mathrm{atm}$. Nous pouvons donc calculer, dans le cas d'échantillons de GaSb de type $\eta$ pour lesquels toutes les impuretés sont ionisées, le taux de variation de la bande (111) par rapport à la bande de valence :

$$
\begin{gathered}
\frac{\mathrm{d} E_{\mathrm{g}_{2}}}{\mathrm{~d} P}=\frac{\mathrm{d} E_{\mathrm{g}_{1}}}{\mathrm{~d} P}+\frac{\mathrm{d} \Delta E}{\mathrm{~d} P} \\
\frac{\mathrm{d} E_{\mathrm{g}_{2}}}{\mathrm{~d} P}=5,5 \times 10^{-6} \mathrm{eV} / \text { atm. }
\end{gathered}
$$

Conclusion. - Nous constatons que la bande de conduction (111) du GaSb sous l'effet d'une pression hydrostatique se déplace par rapport à la bande de valence dans le même sens que la bande $(000)$, mais que le taux de variation est plus faible pour la bande (111). Ce qui est conforme aux résultats trouvés déjà par de nombreux auteurs [9], [11], [14], [16]. Nous montrons toutefois qu'en prenant en compte la variation des masses effectives des deux bandes (000) et (111), ainsi que la variation des mobilités dues à la pression, la correction apportée est relativement faible, de l'ordre de quelques \% si nous comparons nos résultats à ceux de Kosicki par exemple qui a négligé la variation de masse effective et la variation de mobilité dues à la pression.

Remerciements. - Les auteurs remercient le Docteur Gouskov qui leur a fourni les échantillons sur lesquels les mesures ont été effectuées.

\section{BIBLIOGRAPHIE}

[1] Smith (C. S.), Phys. Rev., 1954, 94, 42.

[2] Keyes (R. W.), Solid State Phys., 1960, 11, 158.

[3] Bordure (G.), Thèse d'État, Montpellier, mars 1969.

[4] Robert (J. L.), Solid Stat. Communications, 1969, 7, 143.

[5] Brooks (H.), Adv. Electr. Electrons Phys., 1955, 7,85 .

[6] Bardeen (J.) et Schockifey (W.), Phys. Rev., 1950, 80, 72 .

[7] Seitz (F.), Phys. Rev., 1948, 73, 549.

[8] BeEr (A. C.), Solid State Phys., Suppl. 4, 1963, 109.

[9] Kosicki (B. B.) et Paur (W.), Phys. Rev. Letters, $1966,17,1175$.
[10] MaThieu (H.), Thèse d'État, mars 1969.

[11] Madelung (O.), Phys. of III-Y compounds, Wiley, New York, London, 1964.

[12] Averous (M.) et Bougnot (G.), Phys. Stat. Solid, 1967, 21, 665 .

[13] Harland (H. B.) et Wooley (J. C.), Can. J. of Phys., 1966, 44, 2715.

[14] Sagar (A.), Phys. Rev., 1960, 117, 93.

[15] Averous (M.), Thèse d'État (4 octobre 1969).

[16] Kosicki (B. B.) et Paul (W.), Phys. Rev. Letters, 1966, 17, 246.

[17] MCDougali, et Stoxer, Phil. Trans. Roy. Soc. London, 1938, A 237, 67. 